\title{
1 Noncanonical targeting contributes significantly to miRNA-mediated 2 regulation
}

3

4 Jennifer Y. Tan ${ }^{1}$, Baroj Abdulkarim¹, and Ana C. Marques ${ }^{1}$

6 'Department of Computational Biology, University of Lausanne, Switzerland

7 Corresponding authors: anaclaudia.marques@unil.ch; jennifer.tan@unil.ch. 


\section{$1 \quad$ ABSTRACT}

2 Determining which genes are targeted by miRNAs is crucial to elucidate their

3 contributions to diverse biological processes in health and disease. Most

4 miRNA target prediction tools rely on the identification of complementary

5 regions between transcripts and miRNAs. Whereas important for target

6 recognition, the presence of complementary sites is not sufficient to identify

7 transcripts targeted by miRNAs.

8 Here, we describe an unbiased statistical genomics approach that explores

9 genetically driven changes in gene expression between human individuals.

10 Using this approach, we identified transcripts that respond to physiological

11 changes in miRNA levels. We found that a much smaller fraction of mRNAs

12 expressed in lymphoblastoid cell lines (LCLs) than what is predicted by other

13 tools is targeted by miRNAs. We estimate that each miRNA has a relatively

14 small number of targets. The transcripts we predict to be miRNA targets are

15 enriched in AGO-binding and previously validated miRNAs target interactions,

16 supporting the reliability of our predictions. Consistent with previous analysis,

17 these targets are also enriched among dosage sensitive and highly controlled

18 genes.

19 Almost a third of genes we predict to be miRNA targets lack sequence 20 complementarity to the miRNA seed region (noncanonical targets). These 21 noncanonical targets have higher complementary with the miRNA 3' end. The 22 impact of miRNAs on the levels of their canonical or noncanonical targets is 23 identical supporting the relevance of this poorly explored mechanism of 24 targeting. 


\section{INTRODUCTION}

3 Post-transcriptional regulation by microRNAs (miRNAs) is widespread in 4 eukaryotes (Bartel 2004). These small (20-22 nucleotide) noncoding RNAs guide target recognition by the miRNA-Induced Silencing Complex (miRISC), which in turn leads to transcript degradation or translational repression (Bartel 2004). In animals, loss of function mutations in miRISC proteins (Kataoka et al. 2001; Alisch et al. 2007; Morita et al. 2007; Vasquez-Rifo et al. 2012) or proteins involved in miRNA maturation (Bernstein et al. 2003; Wienholds et al. 2003;

10 Giraldez et al. 2005; Wang et al. 2008) are embryonically lethal. This highlights 11 the importance of post-transcriptional regulation by miRNAs, particularly during early development. In contrast, the phenotypes associated with loss of function mutations in individual miRNAs are often cell/tissue specific, consistent with the spatial and temporally restricted expression of most miRNAs (reviewed in (Bartel 2018)). The penetrance and impact of miRNA loss of function mutations is also highly heterogenous, ranging for example from postnatal lethality (Heidersbach et al. 2013) to increased susceptibility to genetic or environmental stressors (Stadthagen et al. 2013). This heterogeneity is likely a consequence of the diverse functions of genes whose expression is regulated by miRNAs. Given the importance of miRNAs in diverse biological processes in health and disease (Bartel 2018), significant efforts have gone into determining the mechanisms of miRNA targeting and identifying the repertoire of individual miRNA targets (Eulalio and Mano 2015).

In animals, miRNA target recognition often relies on the base pairing between the miRNA seed region (nucleotides 2-8) and target miRNA recognition elements (MREs), frequently found at the target 3' untranslated regions (UTRs) (Bartel 2004). We refer to this miRNA targeting architecture as "canonical".

Over the past decades, a wide array of prediction methods were developed to establish the miRNA targetome (Peterson et al. 2014). These softwares primarily rely on identifying well-established canonical signatures of 
1 miRNA:target interactions, including sequence complementarity, accessibility,

2 location and conservation of MREs, as well as favourable binding energy

3 between miRNA and target (Peterson et al. 2014). Notably, the overlap

4 between predictions made by different softwares is often small (Sethupathy et

5 al. 2006) and fewer than $1 \%$ of predicted miRNA targets are supported by

6 experimental evidence for miRISC binding (Fridrich et al. 2019). These

7 analyses highlight the extent of false predictions and our poor understanding of

8 the properties that underlie efficient targeting and regulation by miRNAs.

9 Machine learning approaches, in particular those guided by well-defined

10 interactions between miRNA and target (McGeary et al. 2019), have the

11 potential to improve computational prediction of miRNA targets (Schafer and

12 Ciaudo 2020). But given the scarcity of large and comprehensive sets of true

13 miRNA targets, the development of such tools is currently restricted.

14 Alternatively, miRNAs targets can be experimentally identified based on the 15 evidence of their interaction with miRISC or that their expression levels change 16 in response to genetic miRNA perturbations. Given the ease to measure the 17 impact of miRNA level changes on gene expression levels transcriptome-wide, 18 the latter approach has been extensively used (Bartel 2018). However, the 19 impact of miRNA perturbation on gene expression is 1) often smaller than the 20 inter-individual variation in transcript levels, suggesting many of these changes 21 are inconsequential (Pinzon et al. 2017); and 2) this approach does not allow 22 the distinction between changes due to miRNA targeting (direct) or miRNA23 driven changes in the levels of gene expression regulators, including 24 transcription factors or RNA binding proteins (indirect) (Thomas et al. 2010).

Cross-liking Immunoprecipitation (CLIP) based approaches have been used to identify transcripts bound by miRISC (reviewed in (Lin and Miles 2019)) in vitro 27 (i.e. (Hafner et al. 2010)) and in vivo (i.e. (Li et al. 2019)). Experimental evidence 28 for AGO-binding greatly increases the accuracy of miRNA target predictions. 29 However not all transcripts bound by miRISC are miRNA targets (Agarwal et al. 30 2015). Furthermore, whereas, as expected, transcripts with reproducible 31 binding are enriched among true targets, they are depleted in lowly abundant 32 transcripts leading to false negative predictions (Wessels et al. 2019). 
1 Despite their low sensitivity, methods like CLASH (Helwak et al. 2013) or miR-

2 CLIP (Imig et al. 2015) have experimentally detected direct miRNA:target

3 interactions and revealed that a sizable fraction of these interactions occurs at

4 regions outside of the 3'UTRs or rely on noncanonical or mismatch containing

5 seed sites (Helwak et al. 2013). High-throughput analysis of affinity binding also

6 supports that some miRNAs can recognize their targets through noncanonical

7 configurations (Becker et al. 2019; McGeary et al. 2019). The extent of

8 noncanonical targeting cannot be assessed using most of the currently

9 available computational prediction tools, further highlighting their limitations.

10 To overcome some of limitations of the current miRNA target prediction

11 methods, we developed an approach that explores genetically driven changes

12 in gene expression between human individuals. This approach allowed us to

13 identify canonical and noncanonical targets that respond to physiological

14 changes in miRNA levels. 


\section{RESULTS}

\section{Identification of miRNA targets in LCLs}

To identify physiologically relevant miRNA-target interactions, we leveraged small RNA sequencing for a panel of 360 lymphoblastoid cell lines (LCLs), derived from healthy individuals of European descent with known genotype (Lappalainen et al. 2013; 1000 Genomes Project et al. 2015). For 62\% ( $=444)$ of all LCL expressed miRNAs, we identified at least one single nucleotide polymorphisms (SNPs) in the vicinity of their corresponding miRNA gene whose changes were significantly (FDR $<0.05$, Methods) associated with mature miRNA levels (Figure 1A, Supplementary S1A). We refer to these variants as miRNA quantitative trait loci (mirQTLs). We reasoned that targets of these miRNAs should be inversely associated with these mirQTLs in trans (Methods), consistent with miRNAs being negative regulators of gene expression. We used polyA-selected RNA-sequencing data in these cells and identified 6325 mRNAs (42.6\% of the 14847 LCL-expressed genes) associated in trans in the inverse direction with at least one miRNA mirQTL ( $n=6430$ joint eQTLs, Methods, Figure 1B,C, Supplementary S1B).

The association between a mirQTL and a mRNA can be a consequence of 1) the mRNA levels being regulated by the miRNA (causal model); 2) the miRNA and mRNA being independently associated with the same variant, for example if the same transcription factor regulates transcription of both the miRNA and mRNA (independent model); 3) the miRNA being regulated by the mRNA (reactive model); or 4) an undetermined mode of regulation (undecided model, Figure 1D)(Wang and Michoel 2017). We used causal inference analysis to distinguish between these different possibilities for each joint eQTL-miRNAmRNA trio. We found that with a false discovery rate of $10 \%$, a tenth $(10.3 \%$, $\mathrm{n}=665$ ) of all associations are mediated by changes in miRNA levels (Figure 1E). In total, we identified around $4 \%$ of all LCL-expressed genes (593 out of 14847 ) to be causal targets for 143 miRNAs. This corresponds to an average 
1 of 5 targets per miRNA (median 2 targets, ranging between 1 and 44) (Figure

2 1F) and 1-2 miRNAs targeting each transcript (Figure 1G, Supplementary Table 3 ST1).

A.

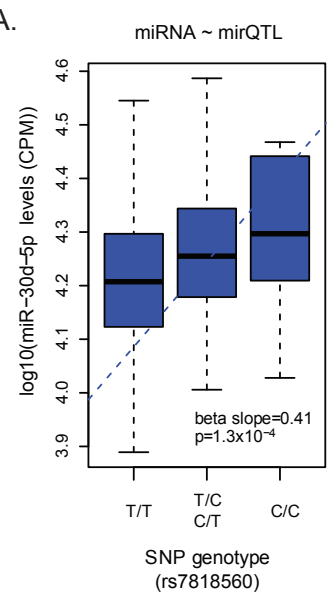

D.

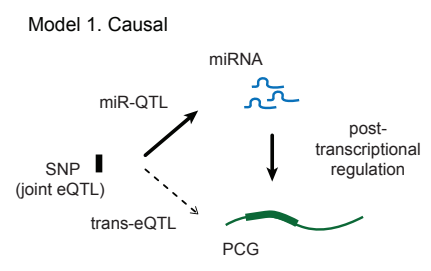

Model 2. Independent

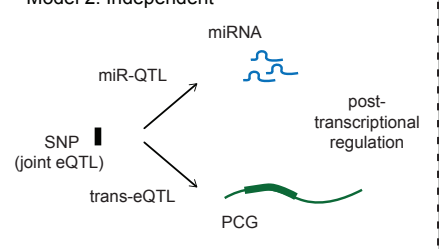

F.

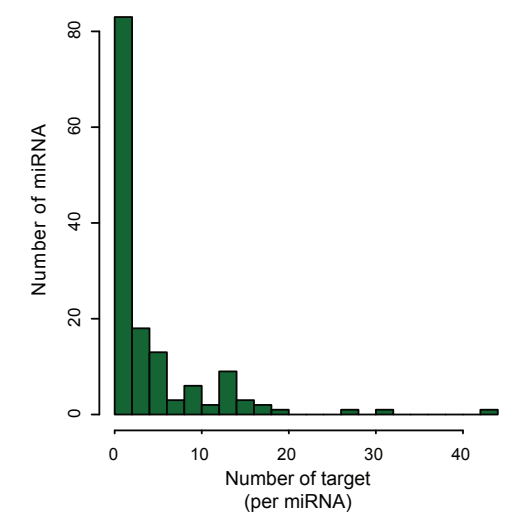

B.

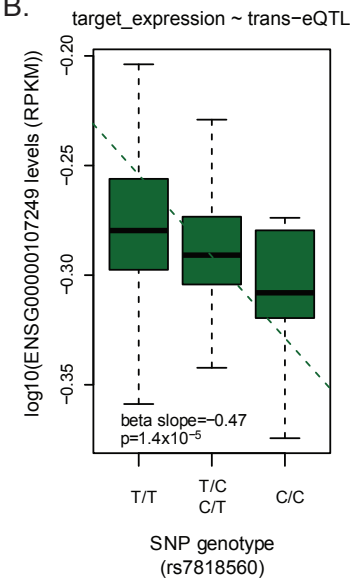

Model 4. Undecided

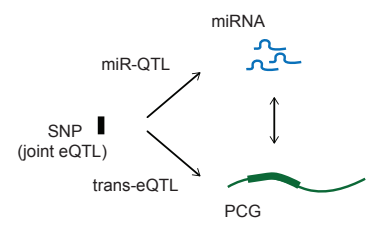

Model 3. Reactive

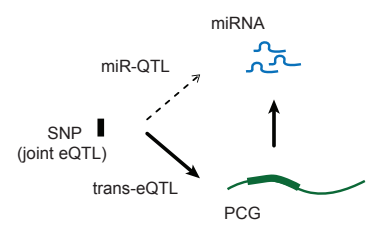

E.
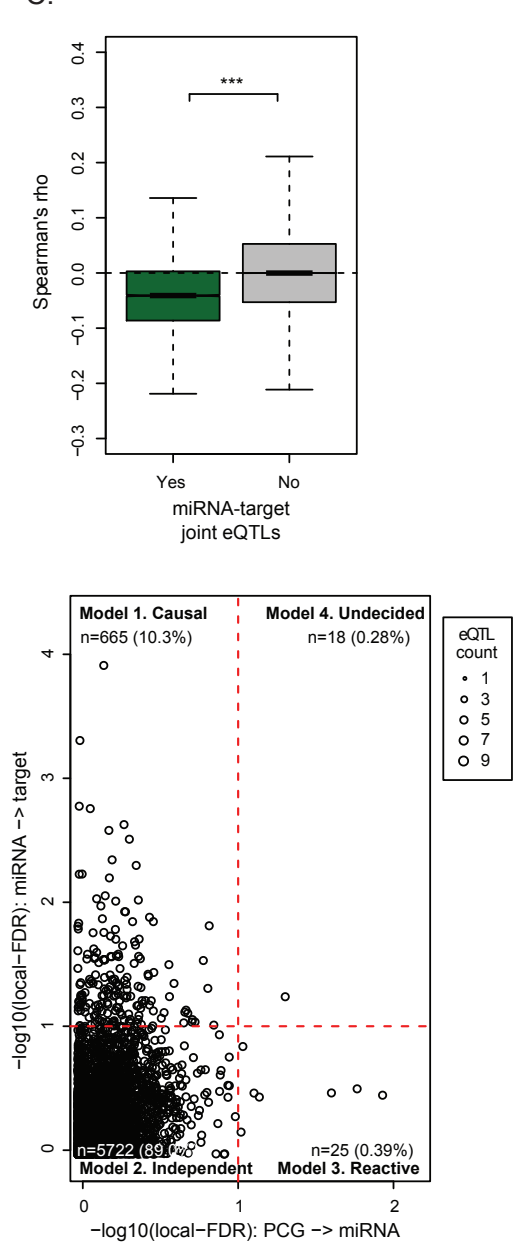

G.

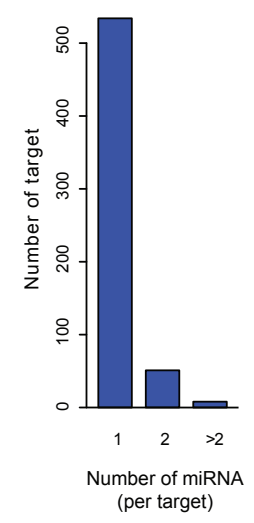

Figure 1. GenVar approach detects physiologically relevant miRNA targets. $(A)$

6 Distribution of miR-30d-5p expression ( $\mathrm{Y}$-axis, CPMs) in individuals with different 7 genotypes of the SNP variant (rs7818560, X-axis, mirQTL). (B) Distribution of GLIS3 
(ENSG00000107249) expression (RPKM, Y-axis) in individuals with different genotypes of the SNP variant (rs7818560, X-axis, trans-eQTL). Beta slope and pvalue of eQTL associations are shown in the inset. (C) The distribution of co-expression (Spearman's correlation) between levels of miRNAs and genes that are jointly (green) or not jointly (grey) associated to the same SNP variant. Differences between groups were tested using a two-tailed Mann-Whitney $U$ test. ${ }^{* * *} p<0.001$. (D) Schematic representation of the four models of causal inference testing that predict the relationship between joint $\mathrm{eQTL}$ variant (black box) associations with the miRNA levels (mirQTL) (blue) and target gene expression level (trans-eQTL) (green): (1) direct association between the variant and miRNA levels mediates the indirect association between that and target gene expression (causal model); (1) the variant is independently associated with miRNA and target levels (independent model); (3) direct association between the variant with target expression mediates the indirect association between that and miRNA levels (reactive model); and (4) the interaction between miRNA levels and target expression is complex (undecided model). Direct associations are depicted as solid lines and indirect associations as dash lines. (E) Scatterplot depicting causal inference testing local FDR associated with each the four models (as illustrated in D). The likelihood of target regulating miRNA levels is plotted on the $x$-axis and the likelihood of miRNA regulation target expression is plotted on the $y$-axis. Number and proportion of joint eQTLs are provided in brackets for each model. Dotted red lines denote significance threshold at local FDR $<0.1$. Frequency distribution of the $(F)$ number of predicted targets per miRNA and $(G)$ number of targeting miRNAs per gene.

Given stronger mirQTL associations might bias towards detecting cases where miRNA levels causally mediate the association between the variant and target expression, we compared the overall strength of associations between mirQTLs and target trans-eQTLs and found no evidence that stronger mirQTLs would favour causal model predictions (Supplementary Figure S1C). Hereafter we refer to transcripts predicted to be targeted by miRNAs based on their genetic variations in humans as GenVar-targets.

\section{Limitations of GenVar-target identification approach}

It is well established that the power to detect quantitative trait loci for relatively lowly expressed genes is limited (GTEx Consortium et al. 2017). We assessed the extent by which this hampered our ability to use the GenVar approach to predict miRNA targets. miRNAs with predicted GenVar-targets $(n=143)$ are among the most highly expressed in LCLs ( $n=716, p=9.4 \times 10^{-7}$, two-tailed Mann- 
1 Whitney $U$ test, Figure 2A). Similarly, we found GenVar-targets are more highly

2 expressed than non-targets $\left(p=6.57 \times 10^{-5}\right.$, two-tailed Mann-Whitney $U$ test,

3 Figure 2B). These findings possibly reflect the approach's limitation to predict

4 lowly expressed miRNAs and target transcripts.

B.

A.

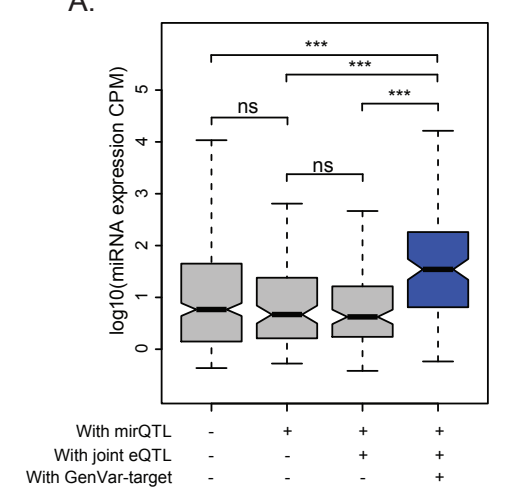

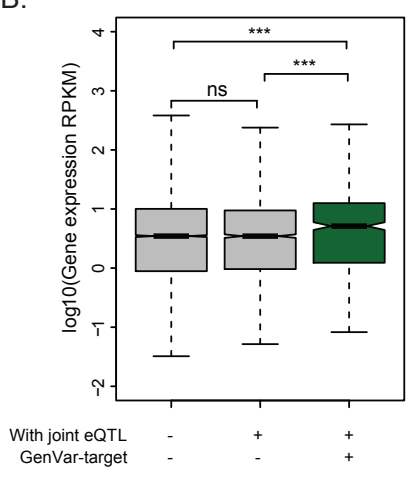

c.

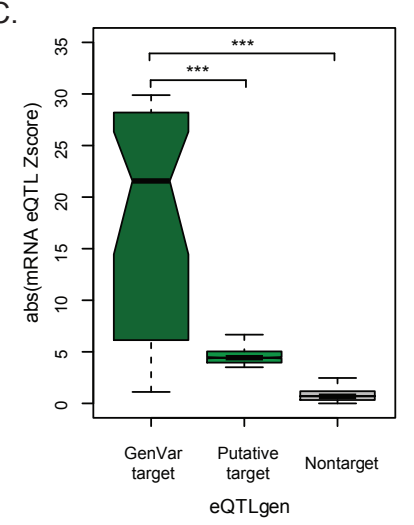

Figure 2. Limitations of the GenVar approach. (A) Distribution of subsets of LCLexpressed miRNA levels with mirQTL associations, whose mirQTLs are jointly associated with target expression (joint-eQTLs), and with predicted GenVar-targets (blue). (B) Distribution of subsets LCL-expressed gene expression levels with those that are jointly associated with a mirQTL (joint-eQTL), and those that are predicted GenVar-targets (green). (C) In blood samples, distribution of trans-eQTL associations (Zscore) between validated GenVar-targets (dark green), putative targets (light green) or nontargets (grey) with mirQTLs. Differences between groups were tested using a two-tailed Mann-Whitney U test. * $p<0.05 ;{ }^{* *} p<0.01$; ${ }^{* * *} p<0.001$; NS $p>0.05$.

Cohort size is also a well-established contributor to the power of quantitative trait analysis (Beavis 1998). To assess whether our predictions were limited by this, we used a dataset that is almost 100 times larger (31,684 blood samples, eQTLGen consortium (Võsa et al. 2018)) than the one used to identify GenVartargets (360 LCLs (Lappalainen et al. 2013)). As the expression levels for mature miRNAs is unavailable for these blood samples and most blood expressed miRNAs are also detected in LCLs (Juzenas et al. 2017), we assumed mirQTLs are conserved between the two cohorts. We used publicly available trans-eQTL data from eQTLgen to assess miRNA-target associations in the blood samples. This data is available for a subset of human variants (GWAS-associated SNPs, $n=10,317$ ) and includes 101 miRQTL variants associated for 23 miRNAs with at least one GenVar-targets in LCLs (total 137 
1 targets). In eQTLgen blood samples, $66 \%$ of GenVar-targets (90/137) were also

2 significantly associated in trans and in the same direction with their respective

3 miRNA, supporting the interaction between them. This high replication rate

4 support robustness of the association between miRNAs and GenVar-targets.

5 Consistent with this, replicated trans-eQTL associations between GenVar-

6 targets and mirQTLs in blood are significantly stronger compared to non-targets

7 in LCLs $\left(p<2.2 \times 10^{-16}\right.$, two-tailed Mann-Whitney $U$ test, Supplementary Figure

8 S2A). Compared to replicated associations between mirQTLs and GenVar-

9 targets, those only found in LCLs (34\%) were similarly associated in blood

$10(p=0.335$, two-tailed Mann-Whitney $U$ test, Supplementary Figure S2A),

11 suggesting some of these are likely explained by LCL-specific miRNA

12 regulation.

13 In addition to the GenVar-targets we validated in blood (validated GenVar14 targets), we found the expression levels of another 48 genes are also correlated 15 in trans with mirQTLs for 9 of the 23 miRNAs (median 1 target per miRNA, 16 ranging from 1 to 22). Moreover, we also detected 470 putative targets for 23 17 miRNAs with no predicted GenVar-targets in LCLs (median 4 targets per 18 miRNA, ranging from 1 to 53). We refer to these 518 genes as putative targets. 19 However, despite their trans-association with mirQTLs, since miRNA levels are 20 not available in eQTLgen, we could not infer the causal relationship between 21 miRNAs and these putative targets. Given in LCLs, GenVar-targets only 22 accounted for a tenth of all genes associated with mirQTLs (Causal model, 23 Figure 1E), we predict only a small portion of these putative targets are 24 regulated by miRNAs. This hypothesis is supported by the significantly stronger 25 trans-associations, in blood, between mirQTLs and validated GenVar-targets 26 compared to putative targets $\left(p<2.2 \times 10^{-16}\right.$, two-tailed Mann-Whitney $U$ test, 27 Figure 2C). Nevertheless, our findings suggest that the small LCL cohort size 28 likely limited our power in comprehensively detecting all miRNA targets. 
1 miRNA GenVar-targets are enriched in AGO-binding and experimentally

2 validated targets

3 Using three widely-used miRNA prediction softwares: TargetScan (Agarwal et 4 al. 2015), RNA22 (Miranda et al. 2006) and miRWalk (Sticht et al. 2018), we 5 estimated between $88 \%$ and $93 \%$ of mRNAs to be targeted by at least 1 of the 6143 GenVar miRNAs (Supplementary Figure S3A). The proportion of genes $7 \quad(n=593)$ we predicted to be targeted by these miRNAs in LCLs is at least 20 8 times smaller (Supplementary Figure S3A). The small number of miRNA targets 9 might not be surprising given the low fraction of miRNA-target predictions common to all the 3 tools $(11-21 \%, n=92812$ out of $474,114,825,295,448,787$

11 total predictions from TargetScan, RNA22, and miRWalk, respectively, 12 Supplementary Figure S3B) and the previously described high rate of false 13 positives associated with the different prediction methods (Fridrich et al. 2019).

14 The extent of these differences and our approach's limitations probed us to investigate how different target prediction sets associate with properties of true miRNA targets. We found that the expression of GenVar-targets is more strongly inversely correlated with the levels of their targeting miRNAs compared to targets predicted by other computational tools in human LCLs (Figure 3A).

Using publicly available iCLIP data in LCLs (Wan et al. 2014), we determined the extent by which the transcripts predicted by the different approaches were bound by AGO2. To minimize the impact of unspecific AGO2 binding in these analyses, we only considered transcripts with experimental evidence of binding across at least two thirds (4 out of 6 ) of the sequenced LCL samples (Methods). We found that GenVar-targets are significantly more often bound by AGO2 $(26.6 \%)$ than genes predicted by the other 3 tools $\left(<17.9 \%, p<2.8 \times 10^{-7}\right.$, twotailed Fisher's exact test, Figure 3B). AGO2-binding density is also significantly higher at GenVar-targets compared to other predicted targets $(p<0.02$, two-

28 tailed Mann-Whitney U test, Figure 3C), supporting the enrichment of GenVartargets in miRISC binding and miRNA regulation. 
A.

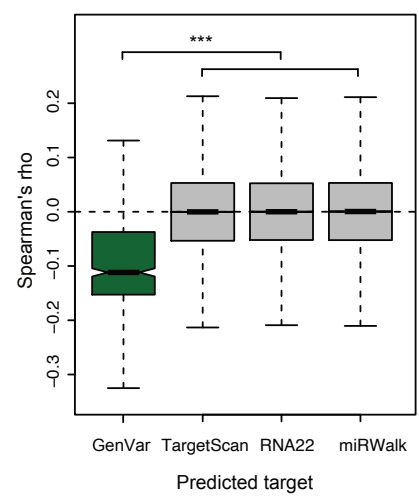

D.

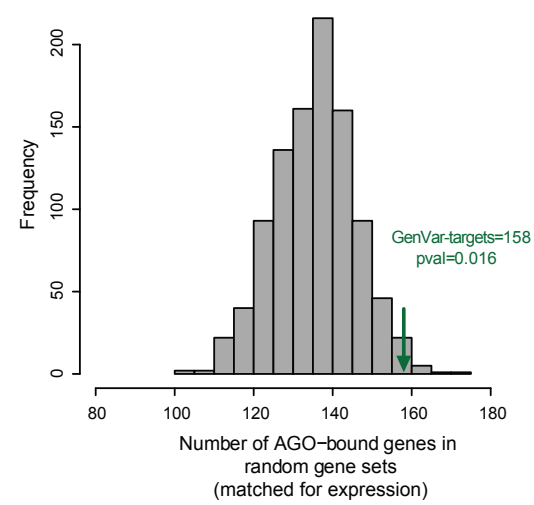

B.

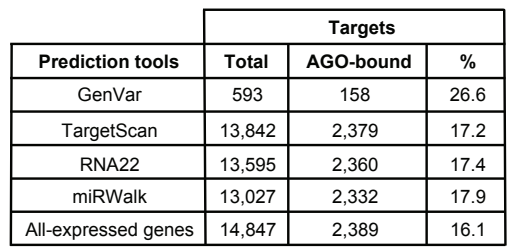

E.

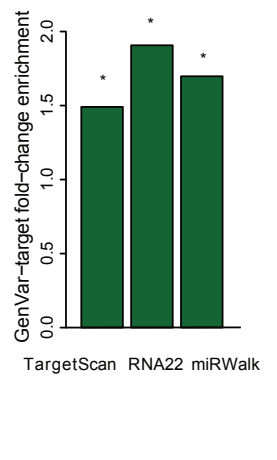

C.

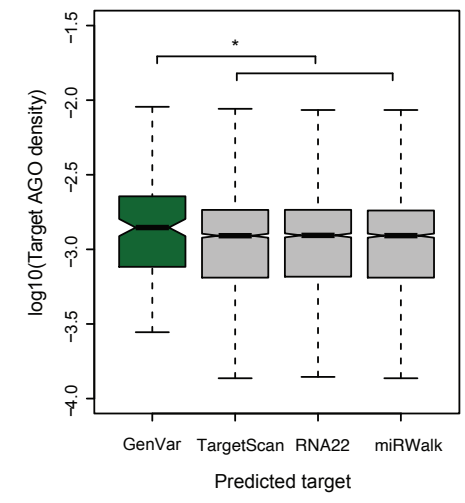

F.

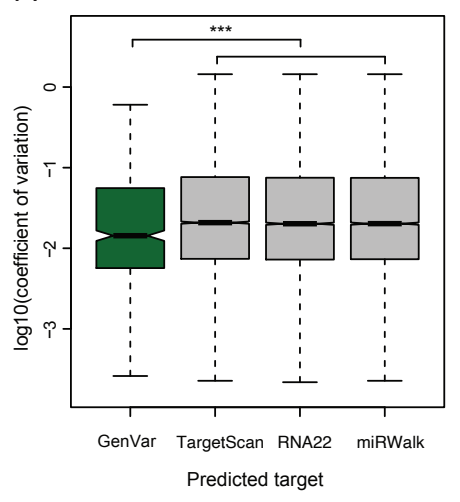

G.

\begin{tabular}{|c|c|c|c|}
\cline { 2 - 4 } \multicolumn{1}{c|}{} & \multicolumn{3}{c|}{ Targets } \\
\hline Prediction tools & Total & Haploinsufficient & $\%$ \\
\hline GenVar & 593 & 100 & 16.9 \\
\hline TargetScan & 13,842 & 1,673 & 12.1 \\
\hline RNA22 & 13,595 & 1,666 & 12.3 \\
\hline miRWalk & 13,027 & 1,633 & 12.5 \\
\hline
\end{tabular}

Figure 3. GenVar targets are a reliable set of miRNA targets. (A) Distribution of coexpression (Spearman's correlation) between miRNA levels and their GenVar-targets (green) or targets predicted by three other tools (TargetScan, RNA22, and miRWalk, grey). (B) Table of the number and proportion of GenVar-targets or targets predicted by other tools to be bound by AGO2. (C) Distribution AGO2 binding density (length of AGO2 binding site/target 3' UTR length) of GenVar-targets (green) and targets predicted by other tools. (D) Distribution of the number of AGO2-bound genes within 1000 sets of randomly sampled genes from other predicted targets with matching expression levels and 3' UTR length as GenVar-targets. Green arrow represent the number of AGO2-bound GenVar-targets. (E) Of all predictions by each of the three prediction tools, the enrichment in the proportion of experimentally validated (using direct Luciferase assays) GenVar-target predictions over nonGenVar-target predictions. (F) Distribution of the coefficient of variation (CV) in expression levels of GenVar-targets (green) and targets predicted by other tools (grey). (G) Table of the number and proportion of target predictions that are annotated as haplo-insufficient. Differences between groups were tested using a two-tailed Mann-Whitney $U$ or twotailed Fisher's exact test. ${ }^{*} p<0.05 ;{ }^{* *} p<0.01 ;{ }^{* * *} p<0.001$; NS $p>0.05$. 
1 As expected, transcripts with detectable AGO2 binding in iCLIP data are in general more highly expressed $\left(p<2.4 \times 10^{-10}\right.$, two-tailed Mann-Whitney $U$ test,

3 Supplementary Figure S4A), reflecting the technical limitations of the 4 technology (Wessels et al. 2019). More than 75\% $(n=447 / 593)$ of GenVar5 targets are less abundant than the median expression level of transcripts with experimental evidence of AGO2-binding ( $n=1101 / 14847$, Supplementary

7 Figure S4B), suggesting that AGO2-binding at many GenVar-targets may have escaped detection due to their relatively low expression. To account for this bias, we compared the extent of AGO2 binding at GenVar-targets relative to randomly sampled expression-matched genes predicted by the other 3 tools and we found that GenVar-targets are significantly more often bound by AGO2 than their counterparts from other prediction methods (1.17-1.19 fold enrichment, $p<0.024$, permutation test, Figure 3D, Supplementary Figure S4CF).

15 We tested the proportion of GenVar-targets that have been experimentally validated. Given the known ascertainment bias to experimentally validate previously computationally predicted miRNA targets, we compared the proportion of experimentally validated predictions by each of the three tools which are also detected using the GenVar approach to those not predicted by GenVar. We found a significantly higher fraction of GenVar-targets ( $p<0.05$,

21 two-tailed Fisher's exact test) has been experimentally validated using direct 22 luciferase assays (1.5-1.9 fold-enrichment, Figure 3E, Supplementary Figure S4G) and in all catalogued experiments (1.4-1.7 fold-enrichment, 24 Supplementary Figure $\mathrm{S} 4 \mathrm{H}, \mathrm{I})$ relative to targets predicted by other tools 25 (Karagkouni et al. 2018), further corroborating that our approach enriches for real miRNA targets.

miRNA-mediated repression on most genes is frequently lower than the interindividual variability in their expression levels (Pinzon et al. 2017). This observation led to the proposal that only the levels of genes whose expression is under tight control, such as haplo-insufficient genes, can be effectively 
1 regulated by miRNAs. Consistent with this, we found that the variation in

2 GenVar-target expression across individuals in the human population are

3 significantly lower compared to target predicted made by other tools $\left(p<9.6 \times 10^{-}\right.$

45 , two-tailed, Mann-Whitney $U$ test, Figure 3F). We also found that GenVar-

5 targets are enriched in haplo-insufficient genes $\left(p<3.0 \times 10^{-3}\right.$, two-tailed Fisher's

6 exact test, Figure 3G), supporting the notion that miRNA-mediated

7 phenotypical changes are likely due to their ability to regulate a relatively small

8 set of dose-sensitive genes (Pinzon et al. 2017; Seitz 2017).

10 Cancer-disrupted miRNA abundance is associated with causal target 11 expression changes

12 Given the limitations in iCLIP technique and the ascertainment bias in reporting 13 of miRNA validation experiments, we sought an alternative unbiased approach 14 to assess the reliability of GenVar-target predictions. We took advantage of 15 extensive genotyping and transcriptomic data from blood-derived cancer 16 samples to assess the impact of changes in miRNA expression on GenVar17 target levels. Specifically, we identified individuals that carry copy number 18 variants (CNVs) that overlap primary miRNA loci in Lymphoid Neoplasm Diffuse 19 Large B-cell Lymphoma (DLBC, Figure 4A)(Cancer Genome Atlas Research et 20 al. 2013). These CNVs resulted in homozygous loss or multiple amplifications 21 of 35 miRNAs (48 samples in total, median 1 samples per CNV, Supplementary 22 Table ST2). 
A.

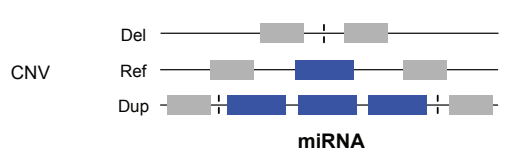

B.

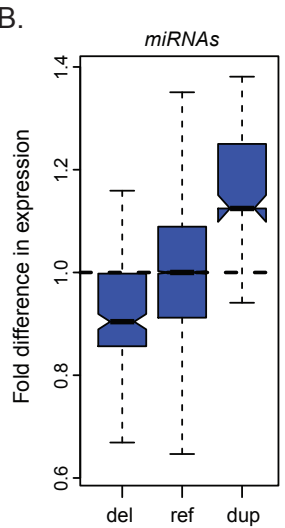

C.

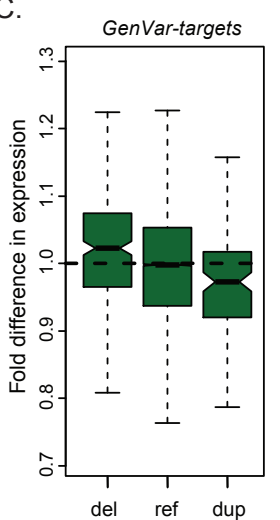

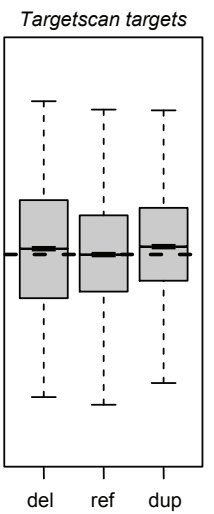

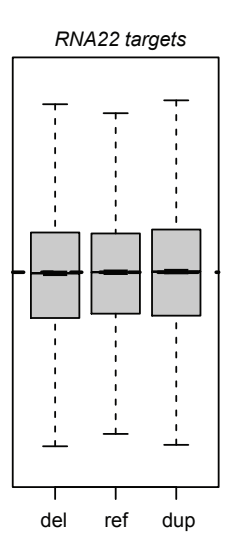

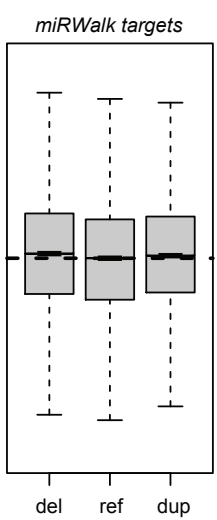

Figure 4. GenVar-target levels are impacted by change in miRNA copy number. (A) Copy number variations in DBLC cancer samples can result in duplications or deletions of primary miRNA loci. (B) Distribution of the fold difference in miRNA levels (CPM, blue), relative to the median of diploid samples, of individuals that carry homozygous deletions, diploid genotype, or multiple duplications at primary miRNA loci. (C) Distribution of fold difference in gene expression levels (RPKM) of GenVartargets (green) or targets predicted by other tools (grey), relative to the median of diploid samples, between individuals that carry homozygous deletions, diploid genotype, or multiple duplications at their targeting miRNAs. Differences between groups were tested using a two-tailed Mann-Whitney $U$ test. ${ }^{*} p<0.05 ;{ }^{* *} p<0.01$; ${ }^{* \star \star} p<0.001 ;$ NS $p>0.05$.

Mature miRNA expression levels corroborated that, as expected, these CNVs led to decreased or increased miRNA expression by at least 9 and $12 \%$, respectively (Figure 4B) relative to individuals that are diploid for the miRNA gene. The relative small impact of copy number changes on miRNA expression are likely a consequence of samples containing mixed normal and tumor cells (Cancer Genome Atlas Research et al. 2013). Changes in miRNA abundance were significantly negatively associated with expression levels of predicted GenVar-targets ( $p<0.05$, two-tailed Mann-Whitney $U$ test) but not with the levels of targets predicted by other tools (Figure 4B,C), substantiating direct miRNA post-transcriptional regulation of GenVar-targets.

In summary, our results support that GenVar-targets are enriched 1) for 
1 AGO2-binding, 2) for experimentally validated miRNA-target interactions, and

2 3) are inversely impacted by changes in disrupted miRNA levels in cancer

3 samples. These observations support that GenVar-targets represent a reliable

4 catalogue of biologically relevant miRNA targets.

6 miRNA targeting is enriched in canonical binding at 3'UTRs

7 Given that our approach does not rely on the identification of perfectly 8 complementary regions to the miRNA seed within transcripts, we next sought 9 to estimate the extent of canonical and noncanonical miRNA targeting (Hausser and Zavolan 2014). Consistent with previous reports (Lewis et al. 2005), we

11 found the majority of GenVar-targets (404 out of 593, 68.1\%) harbour perfect 12 seed matches for their targeting miRNAs at their 3' UTRs (canonical 13 targets)(Figure 5A). This proportion is significantly higher than randomly 14 sampled genes with matching expression level and 3' UTR length $(p=0.001$, permutation test, Supplementary Figure S5A). Compared to other predicted targets that also contain complementary seed regions, alignment profiles of canonical miRNA GenVar-target interactions showed these transcripts bear longer sequence complementary binding sites to miRNA seed region $(p=0.039$, two-tailed Mann Whitney test, Supplementary Figure S5B,C). This is consistent with significantly higher number of 8-mer and 7-mer binding sites found within GenVar-targets (52.5\% 6-mer, 30.2\% 7-mer, and 17.3\% 8-mer) compared to other predicted targets $\left(\mathrm{p}<4.3 \times 10^{-3}\right.$, two-tailed Fisher's exact test, Supplementary Figure S5D).

In addition, some miRNAs have also been found to interact with regions in their target's 5' UTRs or coding sequences (CDSs) (Chi et al. 2009; Helwak et al. 2013). Of the $31.9 \%$ of GenVar-targets $(n=189)$ with no canonical miRNA binding sites in their 3' UTR targets, seed-matching sites were found at their 5' 28 UTR and CDS for 9.0\% $(n=17)$ and 31.2\% $(n=59)$, respectively. Unlike 29 canonical GenVar-targets (Supplementary Figure S5A), these proportions are not significantly different from randomly sampled genes with matching 
1 expression level and length of $5^{\prime}$ UTR or CDS $(p>0.09$, permutation test,

2 Supplementary Figure S5E,F). Our findings are in line with the current

3 understanding that miRNA targeting at $3^{\prime}$ UTR is more likely to lead to

4 physiological changes in target abundance (Gu et al. 2009).

$6 \quad$ Noncanonical targets bear extensive complementarity to miRNA 3' end

7 Apart from perfect matches to the miRNA seed region that form canonical

8 miRNA binding sites, sequence complementarity sites outside the seed region

9 have also been shown to contribute to guiding miRNA target interactions

10 (Becker et al. 2019; McGeary et al. 2019). To investigate binding architecture

11 that underlie noncanonical miRNA targeting, we focused on the $31.9 \%$ of

12 GenVar-targets that lack seed matching miRNA binding sites at 3' UTRs (Figure

$135 \mathrm{~A}$, Supplementary Table ST3). In LCLs, the correlation between miRNAs and

14 their canonical or noncanonical targets is statistically indistinguishable $(p=0.81$,

15 two-tailed Mann-Whitney $U$ test, Figure 5B). Similar impact on canonical and

16 noncanonical GenVar-target expression was also observed as a result of

17 disrupted miRNA levels by CNVs in blood cancer samples ( $p>0.29$, two-tailed

18 Mann-Whitney $U$ test, CNVs disrupted the levels of 34 and 21 miRNA canonical

19 and noncanonical GenVar-targets, respectively, Supplementary Figure S6).

20 These findings support that other features may contribute to determining

21 miRNA-mediated regulation for some miRNAs. Compared to randomly

22 sampled genes with matching expression level and length, noncanonical

23 GenVar-targets were not enriched to contain seed-matching MREs at 5' UTR

24 or CDS ( $p>0.05$, permutation test, Supplementary Figure S7A,B). 
A.

\begin{tabular}{|c|c|c|c|c|c|}
\cline { 2 - 6 } \multicolumn{1}{c|}{} & Total & Canonical & $\%$ & Non-Canonical & $\%$ \\
\hline Targets & 593 & 404 & 68.1 & 187 & 31.9 \\
\hline Interactions & 665 & 447 & 67.2 & 218 & 32.8 \\
\hline
\end{tabular}

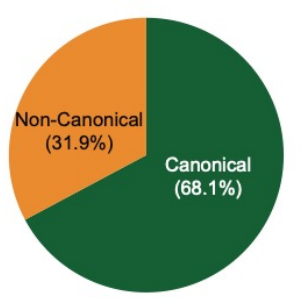

C.

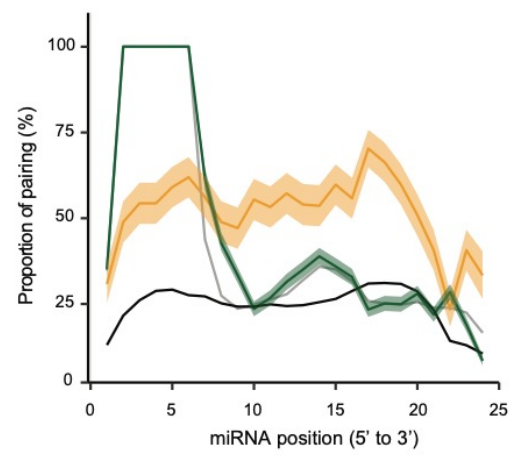

= GenVar-target - canonical MRE = Other-target - canonical MRE EenVar-target - noncanonical MRE $=$ Other-target - noncanonical MRE
B.

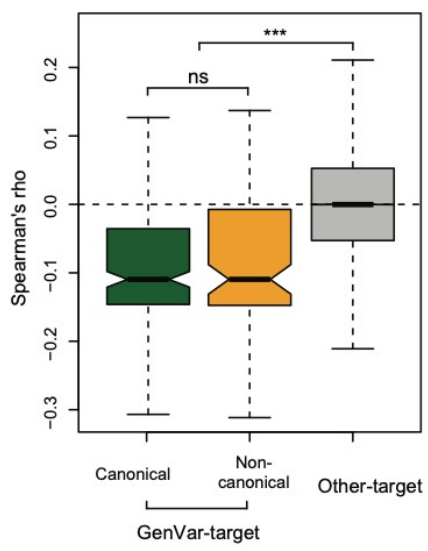

D.

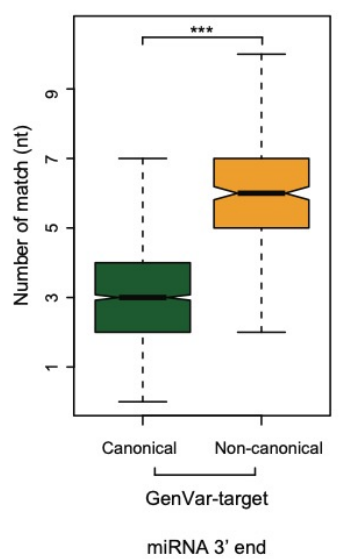

Figure 5. A sizeable fraction of GenVar-targets is noncanonical. (A) Table (Upper panel) and pie chart (lower panel) illustrating the number and proportion of canonical and noncanonical GenVar-targets. (B) Distribution of co-expression (Spearman's correlation) between miRNA levels and their canonical (green) and noncanonical (yellow) GenVar-targets, as well as targets predicted by other tools (grey). (C) Binding profile illustrating the average fraction of complementary sequence alignment between target and miRNA at each nucleotide position across the mature miRNA (5' to 3') for canonical and noncanonical GenVar-targets (green and yellow, respectively), as well as targets predicted other tools that contain canonical and noncanonical binding sites (light and dark grey, respectively). (D) Distribution of the number of complementary sequence alignments (nt) between canonical (green) or noncanonical (yellow) GenVar-targets with 3' end of miRNAs. Differences between groups were tested using a two-tailed Mann-Whitney U test. * $p<0.05 ;{ }^{* *} p<0.01 ;{ }^{* *} p<0.001 ;$ NS $p>0.05$.

We assessed the alignment between noncanonical GenVar-targets and their associated miRNAs. Using adequate alignments between $68 \%$ of target 3 ' UTR and their respective miRNAs, we found each miRNA have on average between 2-3 noncanonical targets (ranging from 1 to 20, Supplementary Figure S7C). Of 
1 all miRNAs with GenVar-targets, $43.3 \%$ regulate gene expression only through

2 the canonical mode of binding, whereas $20.3 \%$ have only noncanonical targets,

3 and $36.4 \%$ have a mixture of both (Supplementary Figure S7D). Alignment

4 profiles of noncanonical miRNA GenVar-target interactions showed that, as

5 expected, complementarity to miRNA seed regions is significantly less frequent

6 than that found for canonical targets $\left(\mathrm{p}<2.2 \times 10^{-16}\right.$, two-tailed Mann Whitney test,

7 Figure 5C, Supplementary Figure S7E). In contrast, extensive nucleotide

8 complementarity was observed between noncanonical targets and 3' end of

9 miRNA ( $p<2.2 \times 10^{-16}$, two-tailed Mann-Whitney test, Figure 5C,D), suggesting

10 imperfect seed interaction may be compensated by additional binding outside

11 of miRNA seed region. 


\section{DISCUSSION}

2 Target identification remains one of the biggest challenges when trying to

3 understand the contribution of miRNAs to post-transcriptional regulation of

4 gene expression.

5 Here we propose a target prediction method that leverages genetically driven

6 changes in miRNA and transcript expression levels within humans to identify 7 miRNA targets (GenVar-targets). Compared to targets predicted by other 8 approaches, GenVar-targets are enriched in AGO2 binding, supporting their

9 frequent association with the miRISC. As expected, given their established role 10 in post-transcriptional repression, miRNA copy number changes in cancer, are 11 inversely correlated with GenVar-target expression. This is in contrast to what 12 we observe for miRNA targets predicted using other approaches, whose expression remain unchanged. Finally, a sizable fraction of GenVar-targets are 14 validated in an independent dataset. These observations support the physiological relevance of our miRNA target predictions and the high specificity, especially relative to available alternatives, of the GenVar approach.

GenVar-targets account for $4 \%$ of mRNAs expressed in lymphoblastoid cell lines (LCLs). The proportion of transcripts we predict to respond to physiological changes in miRNA levels is significantly smaller than what is estimated by other tools. This discrepancy between ours and other estimates is in part explained by the relatively high rate of false positive predictions of other methods [(Fridrich et al. 2019) and supported by our own analysis]. We also analyzed how different factors, including the expression levels of miRNAs, mRNAs and size of the

24 human cohort, impact the sensitivity of the GenVar approach and likely increase false negative predictions. We found that, as expected, miRNA target associations are significantly stronger in larger cohorts. The large cohort size also allowed us to identify an additional set of putative targets not detected using the smaller set of LCL samples. We therefore anticipate that the ongoing trend to expand population cohort size will result in increased prediction 
1 sensitivity and has the potential to extend the repertoire of physiologically

2 relevant miRNA targets.

3 In summary, our analysis supports the functional relevance of GenVar-targets 4 with relatively low false positive predictions. High specificity of GenVar predictions is accompanied by decreased sensitivity that we anticipate can be minimized with increased cohort size. Furthermore, given most miRNAs are tissue specifically expressed, GenVar-targets identified in LCLs are therefore most likely relevant for this and closely related cells/tissues. The application of this approach to other cells or tissues has the potential to uncover the miRNA targetome in different tissues and to reveal potential tissue-specific features of 11 miRNA targeting.

12 We further investigated the miRNA target binding architecture of GenVar predictions. Specifically and given that the GenVar approach does not require prior knowledge of miRNAs binding features, it provides an unbiased target set to investigate the extent of noncanonical targeting by miRNAs. Consistent with previous studies (Chi et al. 2009; Hafner et al. 2010; Helwak et al. 2013), we found a sizable fraction (around $32 \%$ ) of miRNA targets are noncanonical. The impact of miRNA on target expression is independent of binding architecture since variation in miRNA levels was similarly associated with changes in the expression of their canonical or noncanonical targets. Our observations are in

21 line with recent high-throughput binding affinity experiments (Becker et al. 2019;

22 McGeary et al. 2019) and earlier studies (Vella et al. 2004; Didiano and Hobert 23 2006), and support the functional relevance of noncanonical targeting by miRNAs and the diversity of their binding architecture.

This mode of targeting, which we predict accounts for around a third of miRNA target interactions, is missed by most prediction methods and thus remains poorly understood. Specifically, we found that most noncanonical targets sites that lack perfect seed matches contained additional sequence complementarity with 3 ' ends of the miRNA, suggesting mismatches between target and miRNA seed regions can be compensated by additional matches outside the seed. 
bioRxiv preprint doi: https://doi.org/10.1101/2020.07.07.191023; this version posted July 7, 2020. The copyright holder for this preprint (which was not certified by peer review) is the author/funder, who has granted bioRxiv a license to display the preprint in perpetuity. It is made available under aCC-BY-NC-ND 4.0 International license.

1 The availability of this high confidence set of transcripts regulated by miRNAs

2 opens avenue to study the role of additional molecular entities in miRNA 3 regulation.

4 


\section{MATERIAL AND METHODS}

$3 \quad$ Mapping of molecular quantitative trait loci (QTLs)

4 We used miRNA abundance and processed genotype for human EBV-

5 transformed lymphoblastoid cell lines (LCLs) derived from 360 healthy

6 individuals of European descent (CEU, GBR, FIN and TSI) (downloaded from

7 EBI ArrayExpress, accession E-GEUV-1)(Lappalainen et al. 2013). We tested

8 the association between single nucleotide polymorphisms (SNPs) located

9 within the $1 \mathrm{Mb}$ of primary miRNA transcript loci for each of the 715 miRNAs

10 expressed in LCLs with available expression data (Lappalainen et al. 2013).

11 Only SNPs with minor allele frequency (MAF) greater than $5 \%$ were considered 12 in the QTL analyses. miRNA eQTL associations were estimated using FastQTL 13 (version 2.184) (Ongen et al. 2016). To assess the significance of the 14 correlations globally, we first applied multiple testing correction on FastQTL 15 estimated associations by permuting the expression levels of each miRNA 1000 16 times and noted the maximum permuted absolute regression coefficient $\left(r_{\max }\right)$. 17 We considered only miRNA eQTLs with an observed absolute regression 18 coefficient ( $r_{\text {obs}}$ ) greater than $95 \%$ of all permuted $r_{\max }$ values to be significant 19 (Lappalainen et al. 2013). We further performed Benjamini-Hochberg multiple 20 testing correction to estimate FDR $(<5 \%)$ for all SNPs within the $1 \mathrm{Mb}$ vicinity of 21 pri-miRNAs. We identified significant miRNA-eQTLs (mirQTLs) for 62\% ( $n=444)$ of LCL-expressed miRNAs.

The expression levels (RPKM) of protein-coding genes $(n=14,847)$ in the same 360 LCL samples were downloaded from a previous study (as described in (Tan et al. 2017)). We identified trans-eQTLs between all expressed genes and mirQTLs using the same approach as described above with the exception that multiple-testing correction was applied for all trans-eQTL tested. Genes in the vicinity (within $1 \mathrm{Mb}$ ) of pri-miRNAs were not considered in the analysis. Of the 14,847 protein-coding genes expressed in LCLs, we found 6325 genes $(42.6 \%)$ 
1 to be associated in trans and in the inverse direction with mirQTLs for 444

2 miRNA (6430 joint eQTLs).

3 Causality inference between miRNA abundance and target protein-coding

4 gene expression

5 To infer the causal relationship between miRNA levels and putative target gene

6 expression, we considered joint-eQTLs that are associated with both miRNA

7 levels (mirQTL) and their putative target gene abundance (trans-eQTL) (6430

8 joint eQTLs associated with 444 miRNAs and 6325 protein-coding genes). For

9 all such triplets of joint-eQTL - miRNA - target gene expression, we performed

10 causal inference testing using a Bayesian approach as implemented by Findr

11 (Wang and Michoel 2017) by testing the models: (1) the causal model with

12 miRNA where regulates gene expression; (2) the independent model where

13 joint-eQTL variants are independently associated with miRNA levels and gene

14 expression; (3) the reactive model where gene expression mediates miRNA 15 abundance; and (4) the undecided model where causative relationship between 16 miRNA and gene is more complex (Wang and Michoel 2017). Findr was run 17 using the recommended test (findr.pijs_gassist) with default parameters as 18 described in (Wang and Michoel 2017) and significant associations (FDR<10\%) 19 were considered in the analysis.

\section{Assessing sensitivity and replication rate of the GenVar approach}

21 To assess sensitivity of the GenVar approach, we estimated the number of 22 GenVar-targets undetected using LCL samples $(n=360$, 23 Geuvadis)(Lappalainen et al. 2013) in a considerably larger cohort of blood 24 samples ( $n=31,684$ (Võsa et al. 2018)). Given mature miRNA levels are not 25 available in the blood samples, for LCL-identified mirQTL with trans-eQTL data 26 available in blood (64 miRNA, 101 mirQTLs), we considered trans-eQTL 27 associations between all protein-coding genes and these mirQTL to identify 28 additional miRNA targets that are missed in LCLs in blood. We applied multiple 29 testing correction (Benjamini-Hochberg) for the number of trans-eQTL variants 
1 tested and identified additional miRNA GenVar-targets as those with FDR $<5 \%$

2 and are undetected in LCLs.

3 Robustness of trans-eQTL associations identified in LCLs was also assessed

4 in the large cohort of blood samples (Võsa et al. 2018). We considered all trans-

5 eQTL associations between GenVar-targets and mirQTLs tested in blood

6 (association data is available in blood for 23 mirQTL variants for 39 miRNAs

7 with GenVar-targets identified in LCLs). For these trans-eQTLs, we applied

8 multiple testing correction (Benjamini-Hochberg adjusted p-value $<5 \%$ ) for the

9 number of variants tested. We considered associations found in the same

10 direction in eQTLgen blood samples as that found in Geuvadis LCLs to be

11 replicated.

12 Comparison with existing miRNA target prediction tools

13 We downloaded predictions from TargetScan (version 7) (Agarwal et al. 2015),

14 RNA22 (version 2) (Miranda et al. 2006), miRWalk (version 3) (Sticht et al. 2018)

15 and filtered for miRNAs and protein-coding genes expressed in LCLs.

\section{Enrichment in AGO2 binding}

17 We assessed the interaction of GenVar-targets with the miRISC complex using 18 publicly available iCLIP data to identify regions bound by AGO (Wan et al. 2014) 19 (GSE50676). AGO-bound transcripts were downloaded from GSE50676. We 20 only considered AGO binding sites consistently detected in at least two thirds 21 of all replicates (4 out of 6 samples). In total, out of all LCL-expressed genes 22 considered in our analysis $(n=14,847), 18 \%(n=2,669)$ were considered to be 23 robustly bound by AGO.

24 To account for differences in expression levels, LCL-expressed genes 25 predicted as miRNA targets by other tools were divided into two sets of 4 26 equally sized bins based on their expression levels. For each GenVar-target, a 27 gene is randomly selected without replacement from their expression-match 28 gene bins. 


\section{Overlap with experimental validated targets}

2 We used a database containing experimentally validated miRNA target 3 interactions (DIANA-TarBase v8) (Karagkouni et al. 2018) to evaluate the 4 proportion of validated GenVar-targets. We filtered for interactions between 5 LCL-expressed miRNAs and target genes and we considered only interactions 6 validated using all validation experiments $(n=386,278$ interactions) or direct 7 luciferase assays ( $n=190,334$ interactions). To avoid ascertainment bias 8 towards enriched experimental validation of previously computationally 9 predicted targets, when assessing the experimentally validated fraction of

11 from other tools and compared these to predictions from other tools that were 12 not detected by GenVar. 463, 171 and 219 GenVar-targets were also predicted 13 by TargetScan, RNA22, and miRWalk, respectively. Most predictions from 14 other tools were not predicted by GenVar as targets $(474,069,825,124$ and 15448,568 for TargetScan, RNA22, and miRWalk, respectively).

\section{Overlap with haplo-insufficient genes}

17 Haplo-insufficient genes were downloaded from the DECIPHER database 18 (v10)(Firth et al. 2009) and from (Dang et al. 2008). We merged annotations 19 from the two studies. In total, 1754 LCL-expressed genes were found to be 20 haplo-insufficient (1577 from DECIPHER and 288 from Dang et al. 2008).

\section{Impact of genetic variation at miRNA loci on GenVar-target expression}

We considered all copy number variations (CNVs) that overlap primary miRNA transcript loci from blood-derived cancer samples (Lymphoid Neoplasm Diffuse

24 Large B-cell Lymphoma, DLBC) with available genotyping and transcriptomic data (Cancer Genome Atlas Research et al. 2013). We identified CNVs within 48 individuals that led to homozygous loss or multiple amplifications of 35 miRNAs predicted to have at least one GenVar-target (each deep gain or loss event is found in a median of $1 / 48$ samples). CNV data was downloaded from the TCGA Pan-Cancer cohort on the Xena Functional Genomics Explorer 
1 (Goldman et al. 2019). For miRNAs disrupted by CNV, we plotted the average

2 distribution of the fold difference in miRNA levels and their respective target

3 gene expression for each DLBC sample, relative to the median expression of

4 individuals that are diploid for the miRNA gene.

\section{$5 \quad$ Identification of noncanonical miRNA targets}

6 We considered all GenVar-targets with seed-matching (including all 8mer, 7mer 7 and 6mer sites) miRNA recognition elements (MREs) at their 3' UTR, as 8 predicted by the standalone version of TargetScan (version 7) (Agarwal et al. 9 2015), as canonical targets. Comparisons of seed-matching MREs were made to other canonical MRE-containing targets predicted by TargetScan. Seed-

11 matching MREs located at transcript 5' UTR and CDS were predicted using the 12 standalone version of TargetScan (version 7) (Agarwal et al. 2015). Ensembl 13 annotated transcript sequences were used in the analysis (version 98). We 14 tested the enrichment of MRE frequency found at 5' UTR/CDS/3' UTR against 15 that found at 1000 sets of randomly sampled LCL-expressed genes with matching expression level and respective transcript segment length. LCLexpressed genes were divided into two sets of 4 equally sized bins based on their expression levels or length (5' UTR, CDS, or 3' UTR), independently. For each canonical GenVar-target, a gene is randomly selected without replacement from the intersection of their expression-match and length-match 21 gene bins.

22 For noncanonical GenVar-targets, we predicted miRNA binding sites by 23 aligning the full sequence of their targeting mature miRNA with the 3' UTR of 24 target genes using RNAduplex using default parameters with added parameters allowing all alignments within $5 \mathrm{kcal} / \mathrm{mol}$ of the optimal structure to be considered for downstream filtering and GU pairs not considered in the analysis (RNAduplex -e 5 --noGU) (ViennaRNA Package 2.0 (Lorenz et al. 2011)). Alignments that contained consecutive gaps in miRNA sequence or multiple gap openings in target sequence were discarded given the miRISC is 30 unlikely to bind to targets that would result in consecutive bulges in binding 
1 (Brennecke et al. 2005). No restriction was set on the number of mismatches

2 in alignment.

\section{$3 \quad$ Statistical tests}

4 All statistical analyses were performed using the $\mathrm{R}$ software environment for

5 statistical computing and graphics (R Development Core Team 2008). When

6 multiple comparisons were made, we reported the highest $p$-value of all

7 comparisons.

\section{Data Access}

9 Analyses were performed using publicly available command-line tools.

10 Versions and deviations from parameters used are as detailed in the Methods.

11 All scripts used to parse the results are available upon request.

\section{Competing interests}

14 The authors declare that they have no competing interests.

\section{Acknowledgements}

16 We thank members of the Marques group for valuable comments and

17 discussion. We thank Zoltán Kutalik and Olivier Delanau for discussion on 18 population genomics analysis. We thank Constance Ciaudo for discussion on 19 miRNA target validation. This work is funded by the Swiss National Science 20 Foundation grant (PP00P3_179065 to ACM).

\section{Authors' contributions}

22 JYT, BA and ACM designed the study. JYT and BA performed the experiments 23 and analyzed the results. JYT, BA and ACM discussed the results. ACM 24 supervised the study. JYT and ACM wrote the manuscript. All authors approved 25 the manuscript. 
bioRxiv preprint doi: https://doi.org/10.1101/2020.07.07.191023; this version posted July 7, 2020. The copyright holder for this preprint (which was not certified by peer review) is the author/funder, who has granted bioRxiv a license to display the preprint in perpetuity. It is made available under aCC-BY-NC-ND 4.0 International license. 


\section{REFERENCES}

\section{Genomes Project C, Auton A, Brooks LD, Durbin RM, Garrison EP, Kang HM,} Korbel JO, Marchini JL, McCarthy S, McVean GA et al. 2015. A global reference for human genetic variation. Nature 526: 68-74.

Agarwal V, Bell GW, Nam JW, Bartel DP. 2015. Predicting effective microRNA target sites in mammalian mRNAs. Elife 4.

Alisch RS, Jin P, Epstein M, Caspary T, Warren ST. 2007. Argonaute2 is essential for mammalian gastrulation and proper mesoderm formation. PLoS Genet 3: e227.

Bartel DP. 2004. MicroRNAs: genomics, biogenesis, mechanism, and function. Cell 116: 281-297.

Bartel DP. 2018. Metazoan MicroRNAs. Cell 173: 20-51.

Beavis WD. 1998. QTL analysis: Power, precision, and accuracy. In: Paterson AH, editor Molecular dissection of complex traits Boca Raton, FL: CRC Press.

Becker WR, Ober-Reynolds B, Jouravleva K, Jolly SM, Zamore PD, Greenleaf WJ. 2019. High-Throughput Analysis Reveals Rules for Target RNA Binding and Cleavage by AGO2. Mol Cell 75: 741-755 e711.

Bernstein E, Kim SY, Carmell MA, Murchison EP, Alcorn H, Li MZ, Mills AA, Elledge SJ, Anderson KV, Hannon GJ. 2003. Dicer is essential for mouse development. Nat Genet 35: 215-217.

Brennecke J, Stark A, Russell RB, Cohen SM. 2005. Principles of microRNA-target recognition. PLoS Biol 3: e85.

Cancer Genome Atlas Research N, Weinstein JN, Collisson EA, Mills GB, Shaw KR, Ozenberger BA, Ellrott K, Shmulevich I, Sander C, Stuart JM. 2013. The Cancer Genome Atlas Pan-Cancer analysis project. Nat Genet 45: 1113-1120.

Chi SW, Zang JB, Mele A, Darnell RB. 2009. Argonaute HITS-CLIP decodes microRNA-mRNA interaction maps. Nature 460: 479-486.

Dang VT, Kassahn KS, Marcos AE, Ragan MA. 2008. Identification of human haploinsufficient genes and their genomic proximity to segmental duplications. Eur J Hum Genet 16: 1350-1357.

Didiano D, Hobert O. 2006. Perfect seed pairing is not a generally reliable predictor for miRNA-target interactions. Nat Struct Mol Biol 13: 849-851.

Eulalio A, Mano M. 2015. MicroRNA Screening and the Quest for Biologically Relevant Targets. J Biomol Screen 20: 1003-1017.

Firth HV, Richards SM, Bevan AP, Clayton S, Corpas M, Rajan D, Van Vooren S, Moreau Y, Pettett RM, Carter NP. 2009. DECIPHER: Database of Chromosomal Imbalance and Phenotype in Humans Using Ensembl Resources. Am J Hum Genet 84: 524-533.

Fridrich A, Hazan Y, Moran Y. 2019. Too Many False Targets for MicroRNAs: Challenges and Pitfalls in Prediction of miRNA Targets and Their Gene Ontology in Model and Non-model Organisms. Bioessays 41: e1800169.

Friedman RC, Farh KK, Burge CB, Bartel DP. 2009. Most mammalian mRNAs are conserved targets of microRNAs. Genome Res 19: 92-105.

Giraldez AJ, Cinalli RM, Glasner ME, Enright AJ, Thomson JM, Baskerville S, Hammond SM, Bartel DP, Schier AF. 2005. MicroRNAs regulate brain morphogenesis in zebrafish. Science 308: 833-838. 
Goldman M, Craft B, Hastie M, Repečka K, McDade F, Kamath A, Banerjee A, Luo Y, Rogers D, Brooks AN et al. 2019. The UCSC Xena platform for public and private cancer genomics data visualization and interpretation. bioRxiv doi: $10.1101 / 326470: 326470$.

GTEx Consortium, Laboratory DA, Coordinating Center -Analysis Working G, Statistical Methods groups-Analysis Working G, Enhancing Gg, Fund NIHC, $\mathrm{Nih} / \mathrm{Nci}$, Nih/Nhgri, Nih/Nimh, Nih/Nida et al. 2017. Genetic effects on gene expression across human tissues. Nature 550: 204-213.

Gu S, Jin L, Zhang F, Sarnow P, Kay MA. 2009. Biological basis for restriction of microRNA targets to the $3^{\prime}$ untranslated region in mammalian mRNAs. Nat Struct Mol Biol 16: 144-150.

Hafner M, Landthaler M, Burger L, Khorshid M, Hausser J, Berninger P, Rothballer A, Ascano M, Jr., Jungkamp AC, Munschauer M et al. 2010. Transcriptome-wide identification of RNA-binding protein and microRNA target sites by PAR-CLIP. Cell 141: 129-141.

Hausser J, Zavolan M. 2014. Identification and consequences of miRNA-target interactions--beyond repression of gene expression. Nat Rev Genet 15: 599-612.

Heidersbach A, Saxby C, Carver-Moore K, Huang Y, Ang YS, de Jong PJ, Ivey KN, Srivastava D. 2013. microRNA-1 regulates sarcomere formation and suppresses smooth muscle gene expression in the mammalian heart. Elife 2: e01323.

Helwak A, Kudla G, Dudnakova T, Tollervey D. 2013. Mapping the human miRNA interactome by CLASH reveals frequent noncanonical binding. Cell 153: 654665.

Imig J, Brunschweiger A, Brummer A, Guennewig B, Mittal N, Kishore S, Tsikrika P, Gerber AP, Zavolan M, Hall J. 2015. miR-CLIP capture of a miRNA targetome uncovers a lincRNA H19-miR-106a interaction. Nat Chem Biol 11: 107-114.

Juzenas S, Venkatesh G, Hubenthal M, Hoeppner MP, Du ZG, Paulsen M, Rosenstiel P, Senger P, Hofmann-Apitius M, Keller A et al. 2017. A comprehensive, cell specific microRNA catalogue of human peripheral blood. Nucleic Acids Res 45: 9290-9301.

Karagkouni D, Paraskevopoulou MD, Chatzopoulos S, Vlachos IS, Tastsoglou S, Kanellos I, Papadimitriou D, Kavakiotis I, Maniou S, Skoufos G et al. 2018. DIANA-TarBase v8: a decade-long collection of experimentally supported miRNA-gene interactions. Nucleic Acids Res 46: D239-D245.

Kataoka Y, Takeichi M, Uemura T. 2001. Developmental roles and molecular characterization of a Drosophila homologue of Arabidopsis Argonaute1, the founder of a novel gene superfamily. Genes Cells 6: 313-325.

Lappalainen T, Sammeth M, Friedlander MR, t Hoen PA, Monlong J, Rivas MA, Gonzalez-Porta M, Kurbatova N, Griebel T, Ferreira PG et al. 2013. Transcriptome and genome sequencing uncovers functional variation in humans. Nature 501: 506-511.

Lewis BP, Burge CB, Bartel DP. 2005. Conserved seed pairing, often flanked by adenosines, indicates that thousands of human genes are microRNA targets. Cell 120: $15-20$.

Li X, Pritykin Y, Concepcion CP, Lu Y, Rocca GL, Zhang M, Cook PJ, Au YW, Popow O, Paulo JA et al. 2019. High-resolution in vivo identification of miRNA targets by Halo-Enhanced Ago2 Pulldown. bioRxiv doi:10.1101/820548: 820548.

Lin C, Miles WO. 2019. Beyond CLIP: advances and opportunities to measure RBPRNA and RNA-RNA interactions. Nucleic Acids Res 47: 5490-5501. 
Lorenz R, Bernhart SH, Honer Zu Siederdissen C, Tafer H, Flamm C, Stadler PF, Hofacker IL. 2011. ViennaRNA Package 2.0. Algorithms Mol Biol 6: 26.

McGeary SE, Lin KS, Shi CY, Pham TM, Bisaria N, Kelley GM, Bartel DP. 2019. The biochemical basis of microRNA targeting efficacy. Science 366.

Miranda KC, Huynh T, Tay Y, Ang YS, Tam WL, Thomson AM, Lim B, Rigoutsos I. 2006. A pattern-based method for the identification of MicroRNA binding sites and their corresponding heteroduplexes. Cell 126: 1203-1217.

Morita S, Horii T, Kimura M, Goto Y, Ochiya T, Hatada I. 2007. One Argonaute family member, Eif2c2 (Ago2), is essential for development and appears not to be involved in DNA methylation. Genomics 89: 687-696.

Ongen H, Buil A, Brown AA, Dermitzakis ET, Delaneau O. 2016. Fast and efficient QTL mapper for thousands of molecular phenotypes. Bioinformatics 32: 14791485.

Peterson SM, Thompson JA, Ufkin ML, Sathyanarayana P, Liaw L, Congdon CB. 2014. Common features of microRNA target prediction tools. Front Genet 5: 23.

Pinzon N, Li B, Martinez L, Sergeeva A, Presumey J, Apparailly F, Seitz H. 2017. microRNA target prediction programs predict many false positives. Genome Res 27: 234-245.

R Development Core Team. 2008. R: A language and environment for statistical computing. R Foundation for Statistical Computing.

Schafer M, Ciaudo C. 2020. Prediction of the miRNA interactome - Established methods and upcoming perspectives. Comput Struct Biotechnol J 18: 548-557.

Seitz H. 2017. Issues in current microRNA target identification methods. RNA Biol 14: 831-834.

Sethupathy P, Megraw M, Hatzigeorgiou AG. 2006. A guide through present computational approaches for the identification of mammalian microRNA targets. Nat Methods 3: 881-886.

Stadthagen G, Tehler D, Hoyland-Kroghsbo NM, Wen J, Krogh A, Jensen KT, SantoniRugiu E, Engelholm LH, Lund AH. 2013. Loss of miR-10a activates 1po and collaborates with activated Wnt signaling in inducing intestinal neoplasia in female mice. PLoS Genet 9: e1003913.

Sticht C, De La Torre C, Parveen A, Gretz N. 2018. miRWalk: An online resource for prediction of microRNA binding sites. PLoS One 13: e0206239.

Tan JY, Smith AAT, Ferreira da Silva M, Matthey-Doret C, Rueedi R, Sonmez R, Ding D, Kutalik Z, Bergmann S, Marques AC. 2017. cis-Acting Complex-TraitAssociated lincRNA Expression Correlates with Modulation of Chromosomal Architecture. Cell Rep 18: 2280-2288.

Thomas M, Lieberman J, Lal A. 2010. Desperately seeking microRNA targets. Nat Struct Mol Biol 17: 1169-1174.

Vasquez-Rifo A, Jannot G, Armisen J, Labouesse M, Bukhari SI, Rondeau EL, Miska EA, Simard MJ. 2012. Developmental characterization of the microRNAspecific C. elegans Argonautes alg-1 and alg-2. PLoS One 7: e33750.

Vella MC, Choi EY, Lin SY, Reinert K, Slack FJ. 2004. The C. elegans microRNA let7 binds to imperfect let-7 complementary sites from the lin-41 3'UTR. Genes Dev 18: 132-137.

Võsa U, Claringbould A, Westra H-J, Bonder MJ, Deelen P, Zeng B, Kirsten H, Saha A, Kreuzhuber R, Kasela S et al. 2018. Unraveling the polygenic architecture of complex traits using blood eQTL metaanalysis. bioRxiv doi:10.1101/447367: 447367. 
Wan Y, Qu K, Zhang QC, Flynn RA, Manor O, Ouyang Z, Zhang J, Spitale RC, Snyder MP, Segal E et al. 2014. Landscape and variation of RNA secondary structure across the human transcriptome. Nature 505: 706-709.

Wang L, Michoel T. 2017. Efficient and accurate causal inference with hidden confounders from genome-transcriptome variation data. PLoS computational biology 13: e1005703.

Wang Y, Baskerville S, Shenoy A, Babiarz JE, Baehner L, Blelloch R. 2008. Embryonic stem cell-specific microRNAs regulate the G1-S transition and promote rapid proliferation. Nat Genet 40: 1478-1483.

Wessels HH, Lebedeva S, Hirsekorn A, Wurmus R, Akalin A, Mukherjee N, Ohler U. 2019. Global identification of functional microRNA-mRNA interactions in Drosophila. Nat Commun 10: 1626.

Wienholds E, Koudijs MJ, van Eeden FJ, Cuppen E, Plasterk RH. 2003. The microRNA-producing enzyme Dicer1 is essential for zebrafish development. Nat Genet 35: 217-218. 
Figure pioRxiv preprint doi: https://doi.org/10.1101/2020.07.07.191023; this version posted July 7, 2020. The copyright holder for this preprint (which was not certified by peer review) is the author/funder, who has granted bioRxiv a license to display the preprint in perpetuity. It is made available under aCC-BY-NC-ND 4.0 International license.

A.

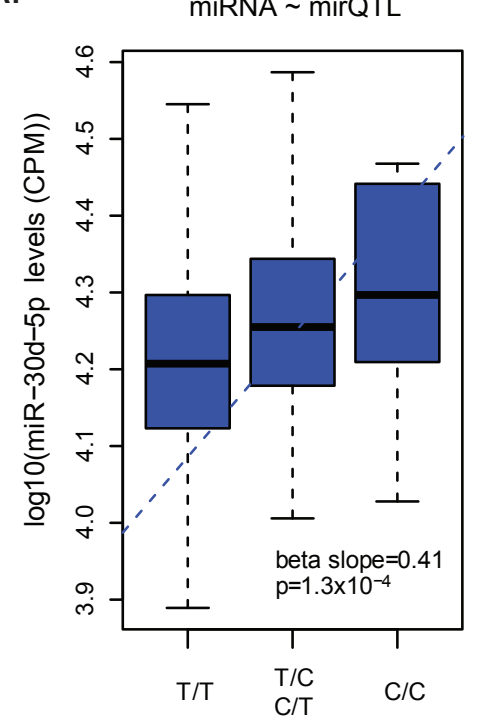

SNP genotype (rs7818560)

D.

Model 1. Causal

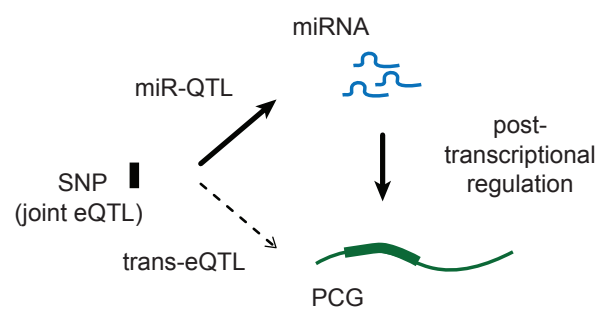

Model 2. Independent

miRNA

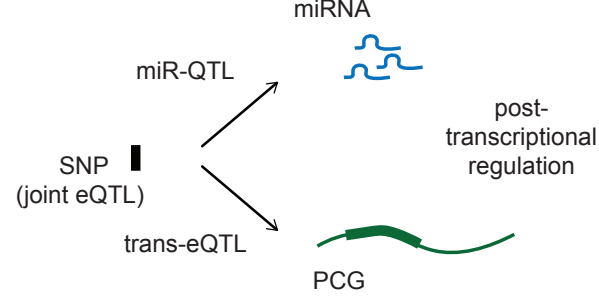

F.

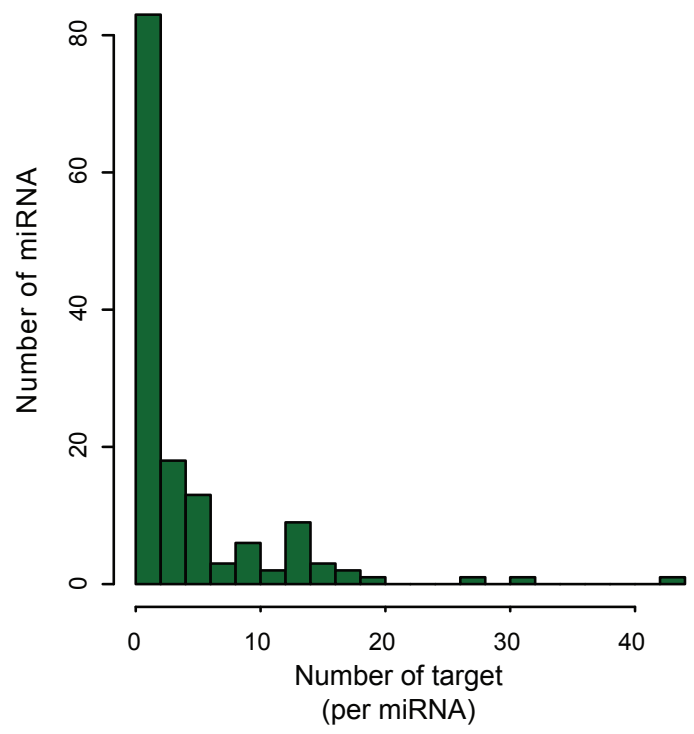

B.

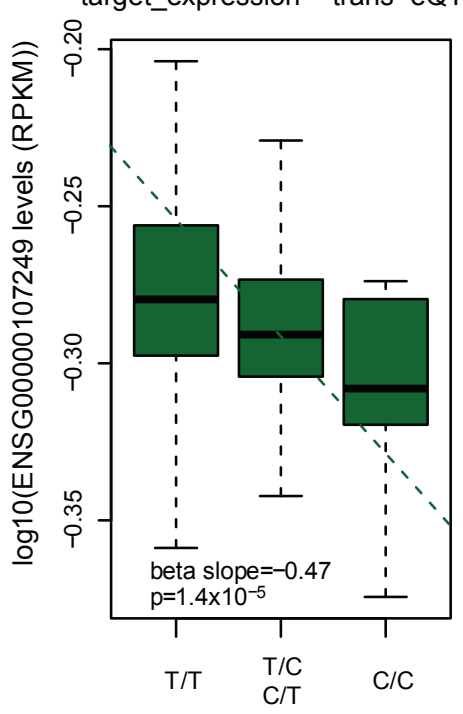

SNP genotype (rs7818560)

Model 4. Undecided

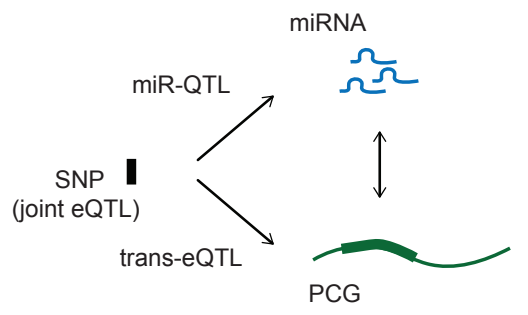

Model 3. Reactive

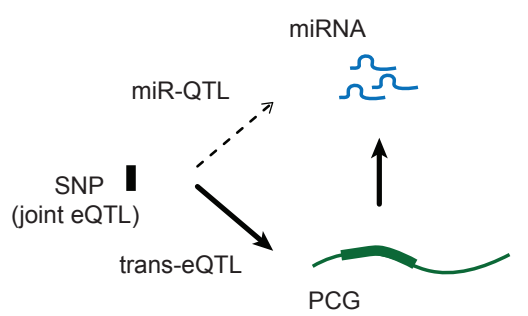

G.

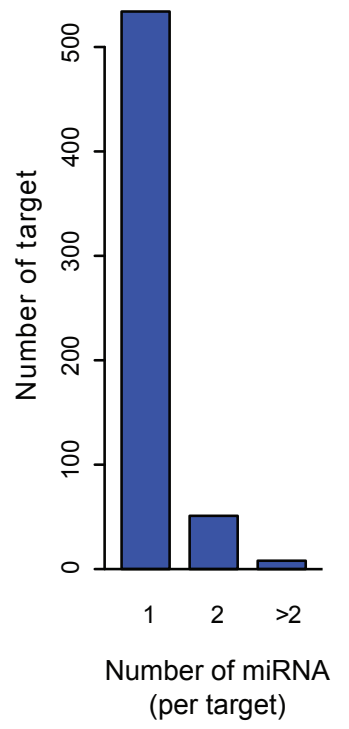

C.

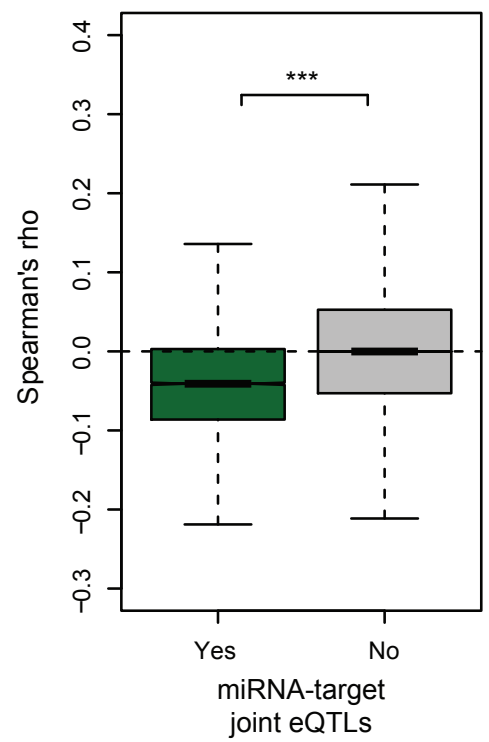

E.

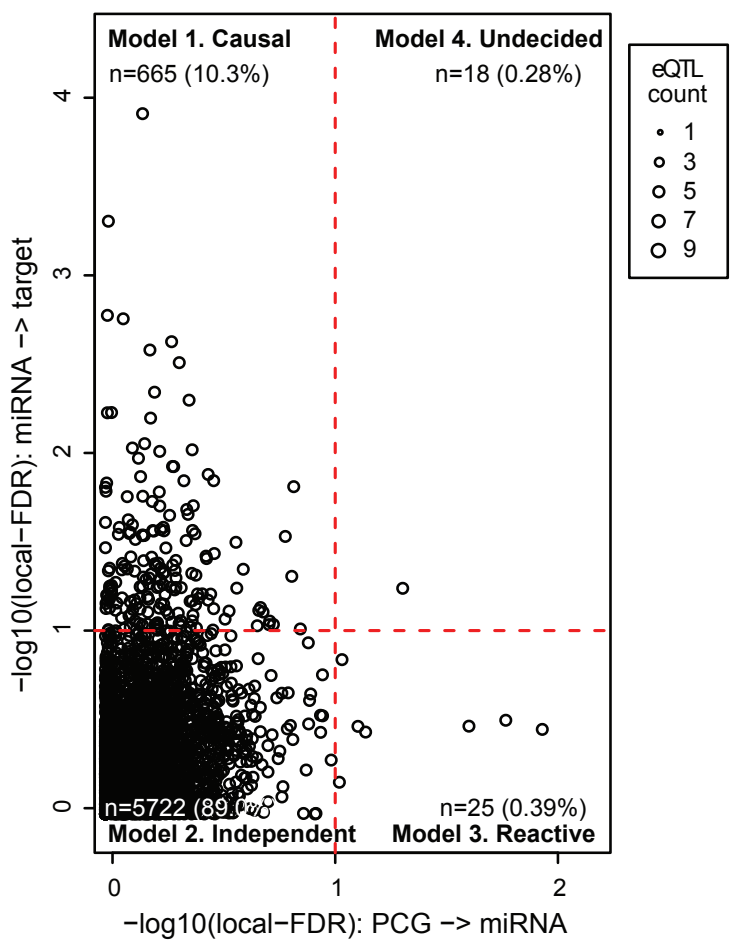


Figure bioRxiv preprint doi: https://doi.org/10.1101/2020.07.07.191023; this version posted July 7, 2020. The copyright holder for this preprint ew) is the author/funder, who has granted bioRxiv a license to display the preprint in perpetuity. It is

A.

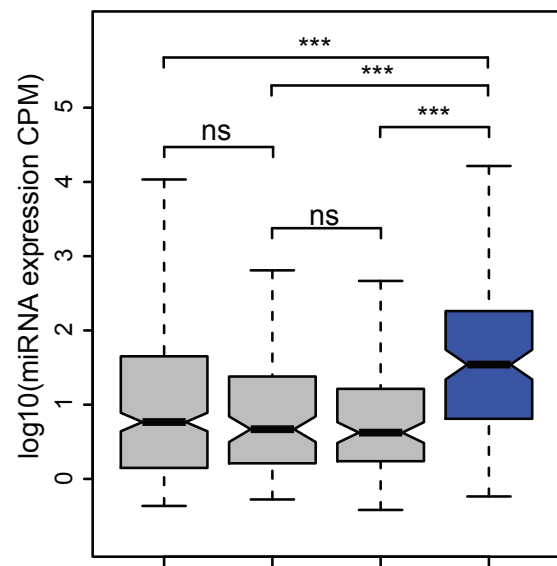

With mirQTL

With joint eQTL With GenVar-target
B.

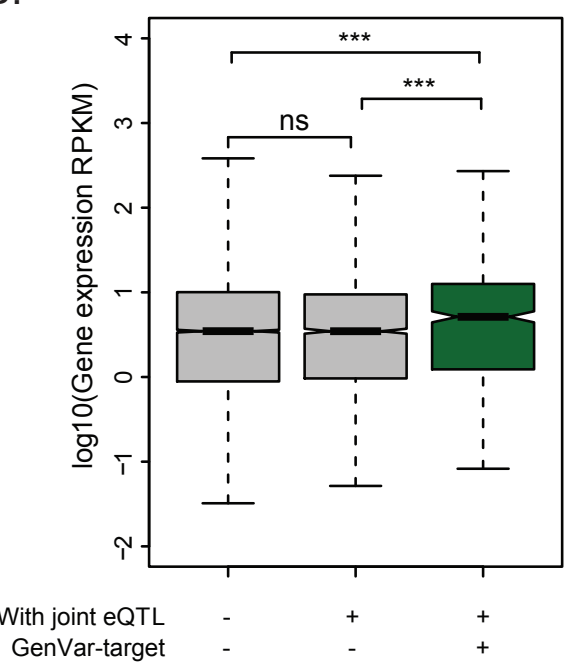

C.

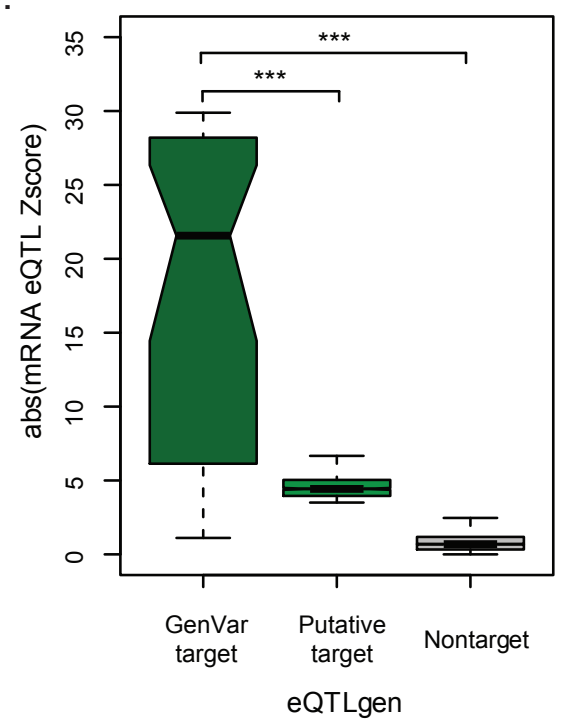


Figure pioRxiv preprint doi: https://doi.org/10.1101/2020.07.07.191023; this version posted July 7, 2020. The copyright holder for this preprint made author/funder, who has granted bioRxiv a license to display the preprint in perpetuity. It is

A.

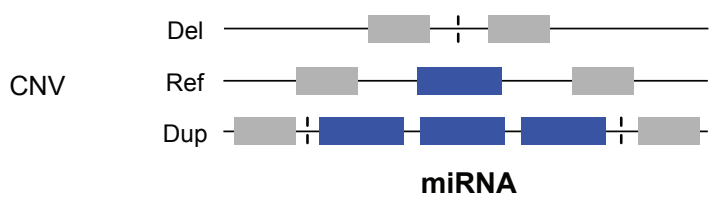

B.

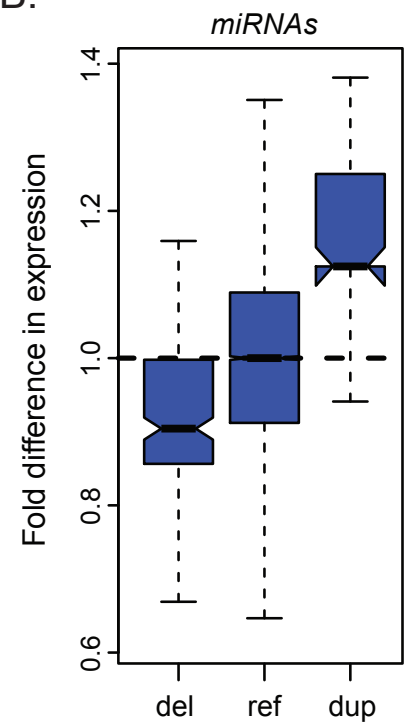

C.

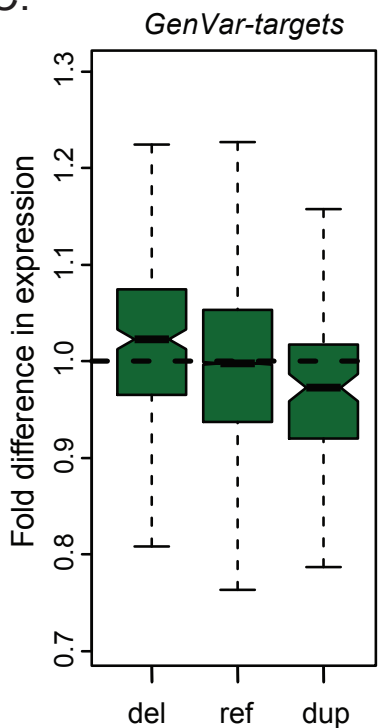

Targetscan targets

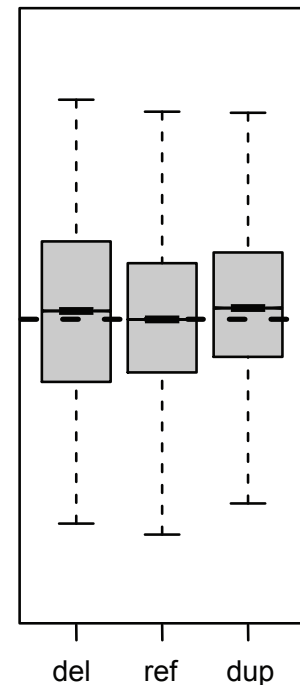

RNA22 targets

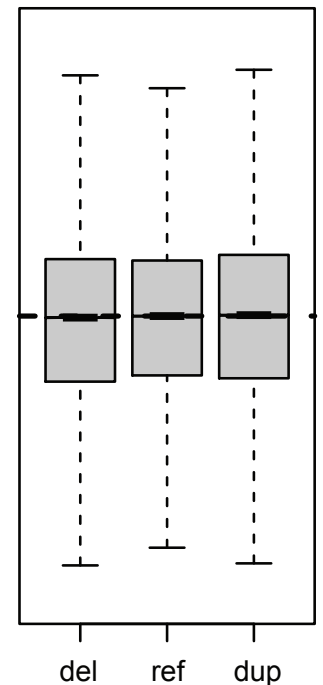

miRWalk targets

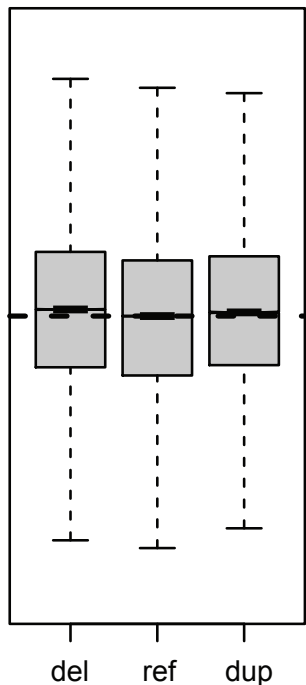


Figure bioRxiv preprint doi: https://doi.org/10.1101/2020.07.07.191023; this version posted July 7, 2020. The copyright holder for this preprint Which was not certified by peer review) is the author/funder, who has granted bioRxiv a license to display the preprint in perpetuity. It is made available under aCC-BY-NC-ND 4.0 International license.

A.

\begin{tabular}{|c|c|c|c|c|c|}
\cline { 2 - 6 } \multicolumn{1}{c|}{} & Total & Canonical & $\%$ & Non-Canonical & $\%$ \\
\hline Targets & 593 & 404 & 68.1 & 187 & 31.9 \\
\hline Interactions & 665 & 447 & 67.2 & 218 & 32.8 \\
\hline
\end{tabular}

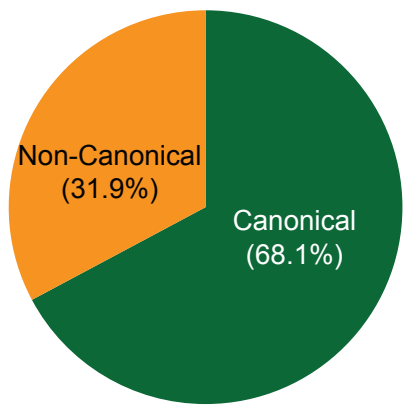

C.

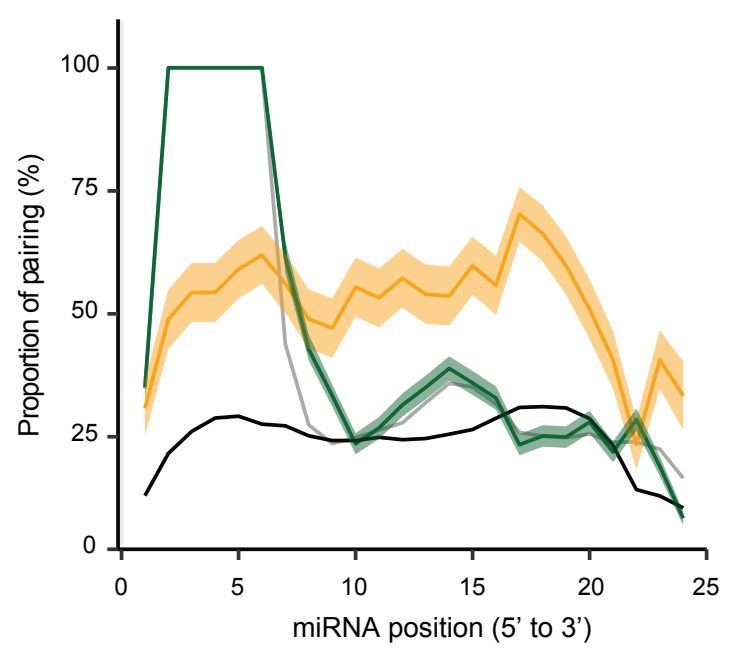

- GenVar-target - canonical MRE - Other-target - canonical MRE - GenVar-target - noncanonical MRE E Other-target - noncanonical MRE
B.

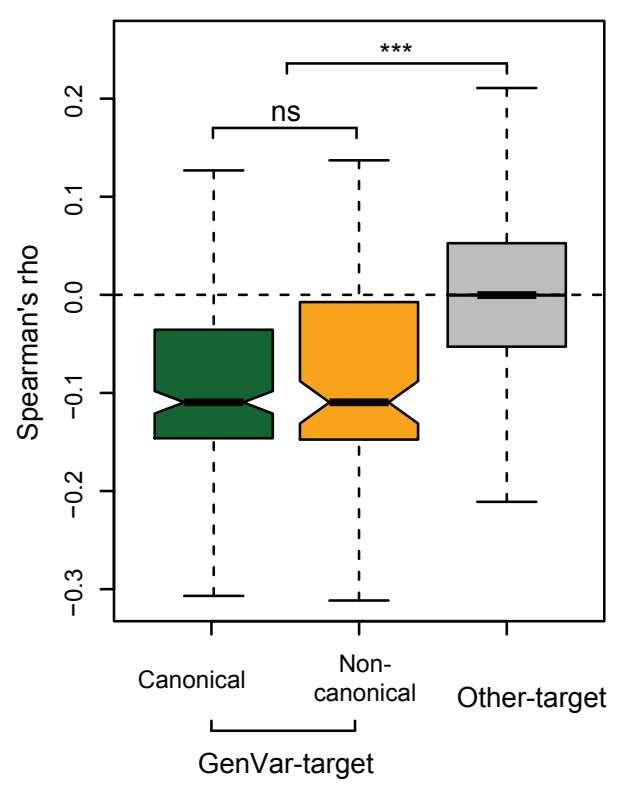

D.

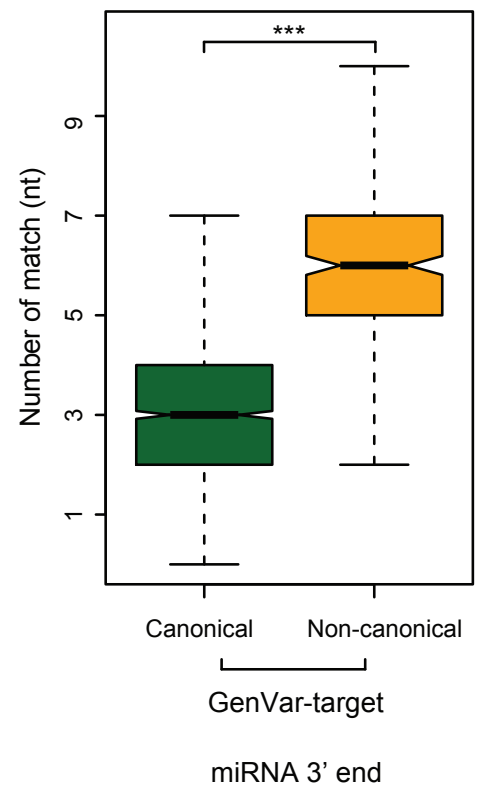

